\title{
Determination of the mixed-mode fracture energy of elastomeric structural adhesives: evaluation of debonding buckling in fibre-metal hybrid laminates
}

\author{
J. C. SuÁrez, F. López, S. Miguel, P. PINIlla and M. A. herreros \\ G.I. Hybrid Materials (HyMeS) Universidad Politécnita de Madrid. ETS Ingenicros Navales, Avda. Arco de La Vitoria, s/n, 2So+o Madrid, Spain
}

ABSTRACT Fibre-metal hybrid laminates combine layers of metal with laminates made of composites - polymeric matrix reinforced with glass-fibre woven fabric. Interface behaviour plays a fundamental role in the overall properties of the hybrid material, especially in the failure mode by debonding buckling of the outermost metal layer. A proper measurement of adhesive fracture energy is required so as to avoid this early failure mechanism during bending. Tapered double cantilever beam test and dissimilar mixed-mode bending test have been used in obtaining mode I and II contributions to the adhesive fracture energy. Data reduction for elastomeric adhesives has been modified in order to account for the variation in compliance during the test due to nonlinear behaviour of the material.

Keywords adhesive; DMMB; debonding buckling; fracture; hybrid material; TDCB.

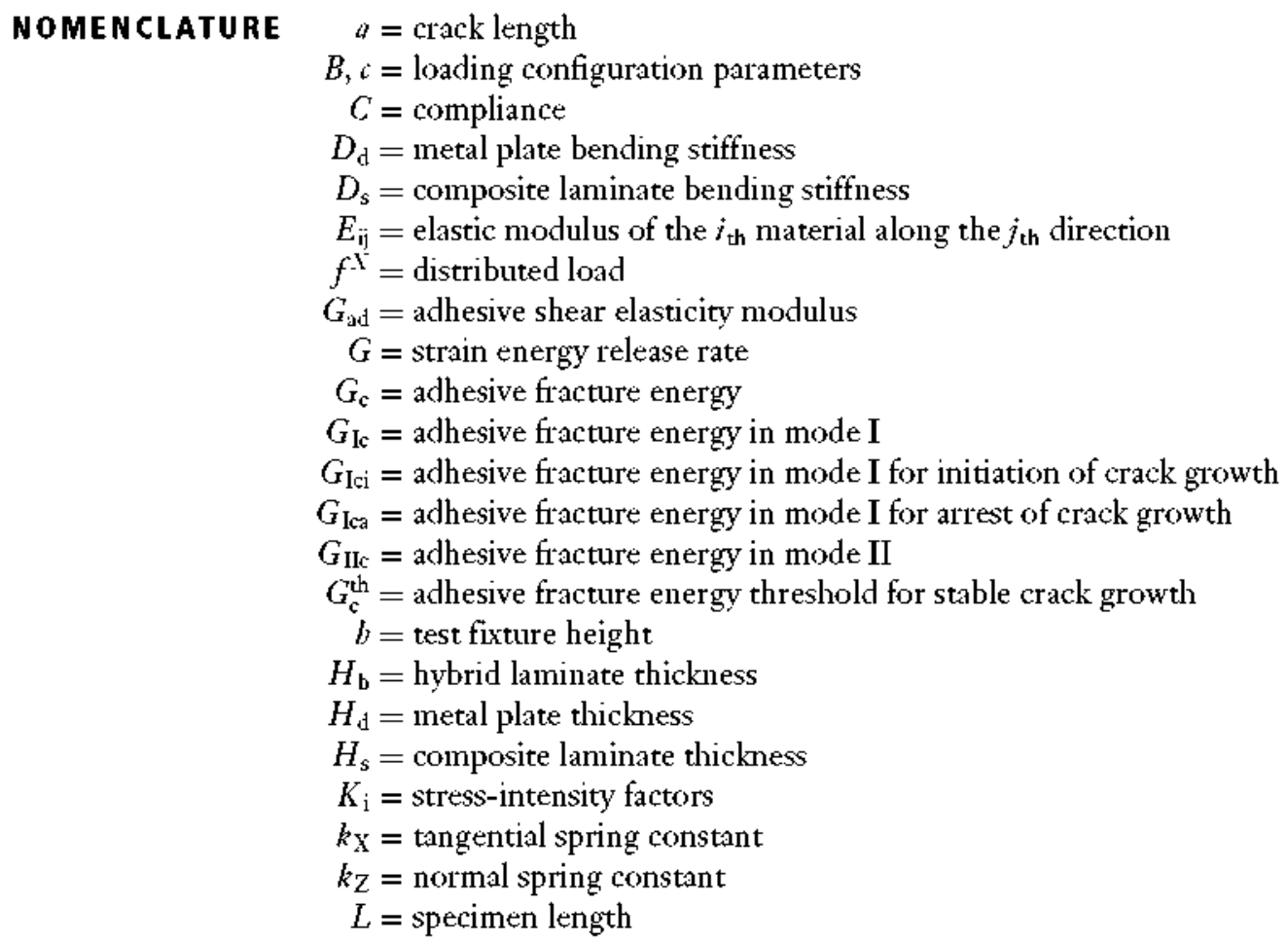




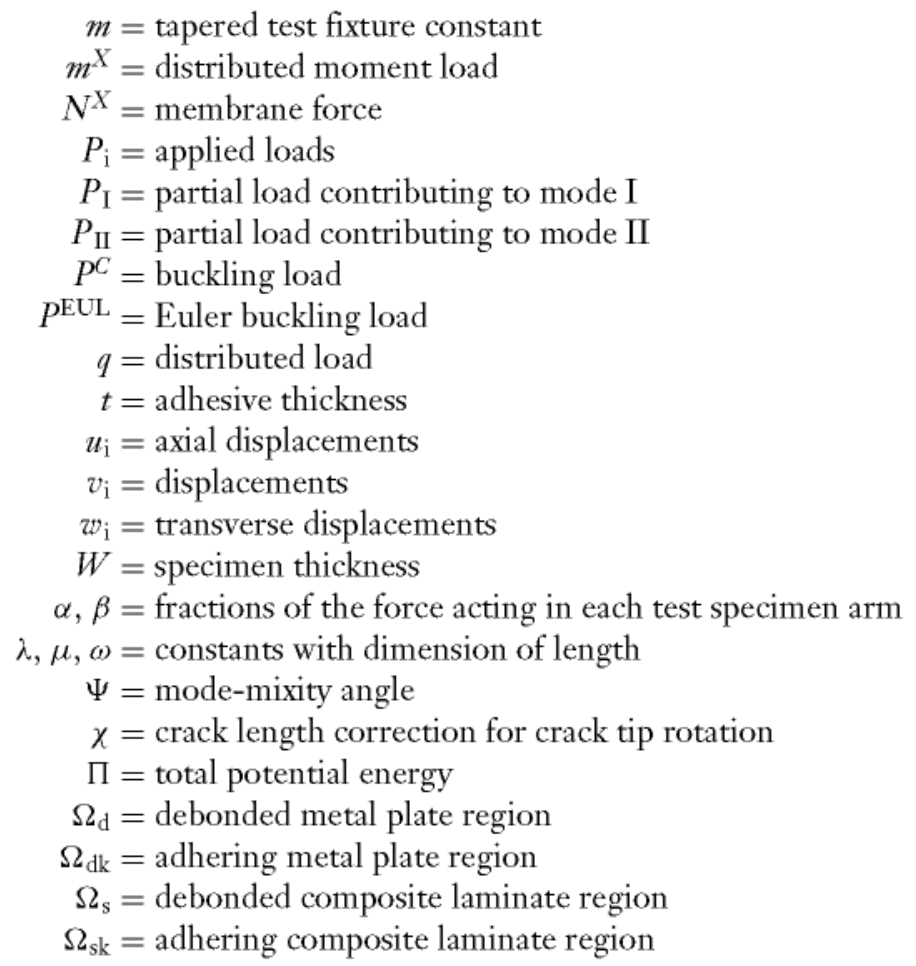

\section{INTRODUCTION}

Critical value for adhesive fracture energy $\left(G_{\mathrm{c}}\right)$ is important so as to characterize the behaviour of structural adhesives from different points of view. Focussing on research and development, $G_{\mathrm{c}}$ is useful in determining the influence on performance of variations in adhesive composition, primers, surface treatment of adherends, carriers, processing, and environmental effects. Considering fitness-for-service evaluation, $G_{\mathrm{c}}$ is used for assessing the capacity of adhesive systems when performing a certain task with a pre-established stress, as it consequently allows an admissible size of defect. $G_{\mathrm{c}}$ has also been the main parameter in developing acceptance criteria for quality control in manufacturing, but such only occurs when there exists a solid base that allows establishing an admissible $G_{\mathrm{c}}$ threshold.

Our interest has been sparked by the study of metal to composite bonded joints in fibre-metal laminates for shipbuilding. ${ }^{1}$ An analytical model ${ }^{2}$ for studying the mechanism of failure in such types of material shows that debonding the buckling (see Fig. 1) of the external metal sheet during bending is very sensible to the actual value of the adhesive fracture energy in the polymer-metal joint. The correct measurement of adhesive fracture energy is then considered relevant for an efficient design of structural hybrid materials.
Application of the tapered double cantilever beam (TDCB) test shows some advantages ${ }^{3}$ that have been applied $^{4}$ in determining critical value in the adhesive fracture energy in mode I, $G_{\mathrm{Ic}}$, more common: (a) height taper allows testing joints made of very low-yield stress materials without producing plastic deformation in the adherends; (b) the rate of change of specimen compliance is independent from crack length. The reduction of data could be done following ASTM D3433 $99^{5}$ by applying corrected beam theory to include shear effects.

A series of effects as proven by Blackman ${ }^{3}$ are not conveniently dealt with in this ASTM standard, that is why,

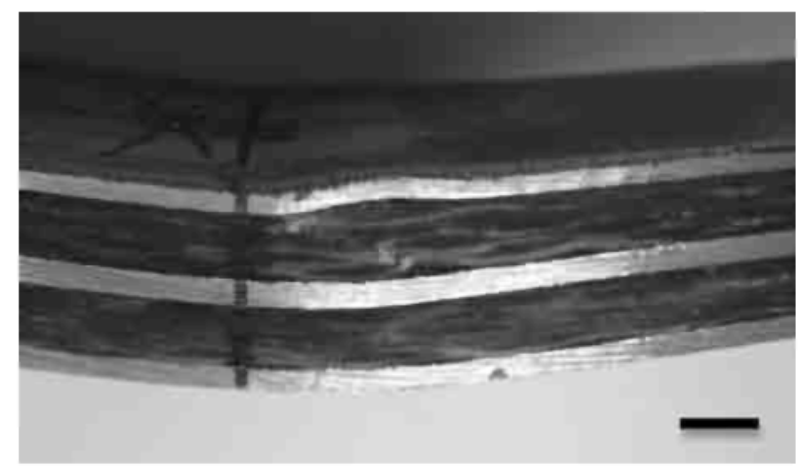

Fig. 1 Debonding buckling in a fibre-metal hybrid laminate (bar $5 \mathrm{~mm}$ ). 
a new protocol has been developed for TDCB testing of metallic material and composites ${ }^{6}$ by the European Structural Integrity Society's Technical Committee 4 of Polymers, Adhesives and Composites (ESIS TC4). The new protocol specifically includes the effect of beam root rotation using an analytical correction factor, which can be considered as an increase in crack length. ${ }^{7}$ Moreover, a correction for system compliance and a validation checking of data after test have also been done.

An international round-robin test ${ }^{4}$ has proven the consistency of measurements obtained by different laboratories. It was proposed and published as British Standard. ${ }^{8}$

This standard procedure is based on the compliance variation of the specimen with crack length, calculated by using standard beam theory or its improved version. Results are acceptable for joints using thin layers of brittle adhesive, but they become increasingly invalid as the assumptions imposed for obtaining analytical expressions are not fulfilled. This is especially true when testing thicker layers of elastic adhesive, where there are large nonlinear strains before the onset of crack extension. In this paper, a modified TDCB specimen designed to be best suited for studying adhesively bonded thin sheet-adherend materials is presented. Metal-to-metal bonded joints using a two-component polyurethane adhesive have been tested at a constant cross-head displacement rate for measuring fracture energy release rate in mode $I$. The load is applied to a specimen until the crack begins to grow rapidly. The load is then stopped. When the load has levelled off at an approximate constant value (the crack has stopped growing), the following values are determined: load to start crack, load when crack stops, and distance from loading end of specimen to the stationary crack tip. The standard test method also specifies that fracture toughness, $G_{\text {Ic }}$ (from load to start crack) and fracture toughness, $G_{\text {Ia }}$ (from arrest load) have to be calculated. As for design purposes, $G_{\mathrm{Ia}_{\mathrm{a}}}$ value is used because it is lower than $G_{\mathrm{Ic}}$, which lies on the safe side.

Calculation of $G_{\text {Ic }}$ using standard expressions, and measuring crack arrest load and crack length for every propagation, has shown that the value is not independent from its crack length. However, data recorded during the tests can be used in modifying results according to the actual compliance of the specimen along the test. In such manner, data reduction yields a crack length independent value of $G_{\mathrm{Ic}}$, which is supposed to be expected from real properties of a polymer.

A more realistic crack-growth criterion should account for mode mixity by assigning different weights to contributions of modes I and II. A rough approach is to consider that only one of the two modes is relevant, and inter-facial crack grows when $G_{\mathrm{I}}=G_{\mathrm{Ic}}$ or $G_{\mathrm{II}}=G_{\mathrm{II}}$. The actual situation states that delamination growth proceeds under mixed-mode condition, and critical energy release rate is a constant to be determined by experimentation, for several ratios of the relative amount of mode $I I$ to mode $I$ at the crack tip. The dissimilar mixed-mode bending (DMMB) test is applied in order to measure the critical energy release rates needed for the inter-facial crack growth under mixed-mode loading for hybrid fibre-metal laminates.

\section{An elastic-interface model for debonding buckling in hybrid fibre-metal laminates}

The literature on debonding buckling is very extensive. Kachanov" and Chai ${ }^{10}$ introduced 'Thin Film Model (TFM) and Thick Column Model (TCM)'. Thereafter, many theoretical and experimental studies have been carried out and a number of cases analyzed, differing in plate geometry, boundary conditions, shape and position of the debonding or delamination. Many questions, such as anisotropy, interface modelling, inter-facial crack nucleation, mixed-mode crack growth and so on are still considered as current research topics.

In debonding buckling processes, the phenomena of instability and fracture are closely related and simultaneously take place; furthermore, a variating formulation is used to derive both the equilibrium solution and the crack growth from a unique, suitably defined functional. ${ }^{11}$ However, the prevailing approach analyses the two concepts separately: first, nonlinear equilibrium problem is solved, and second, a crack-growth criterion is applied. Stability problems can be dealt with in the framework of elasticity theory. Such advantage of this approach is taking into account the inter-laminar stresses, which are responsible for crack growth, as a part of the solution. However, it requires considerable analytical difficulty, even resorting to numerical methods. That is why most studies are based on structural theories. Delaminated plate is modelled as an assemblage of beams and plates, and there is no loss of information about inter-laminar stresses.

As far as the fracture phenomenon is concerned, local approach parameters describing the singularity of the stress field at the delamination front, such as stress-intensity factors $K_{\mathrm{i}}$, is obtained directly when post-critical solution is found via elasticity theory or, alternatively, estimated a posteriori from the computed solution when a structural model is used. Global approach parameters, such as the energy release rate, $G$, can also be obtained. A numerical solution is usually needed for mixed-mode crack-growth criterion in the case of hybrid fibre-metal laminates, where fracture toughness is greater in mode II (sliding) than in mode $\mathbf{I}$ (opening). ${ }^{12}$

A more detailed description of the process of debonding can be achieved through the theory of interface models. In this case, the hybrid laminated plate is schematized a stacking of laminate bound together by interposed 


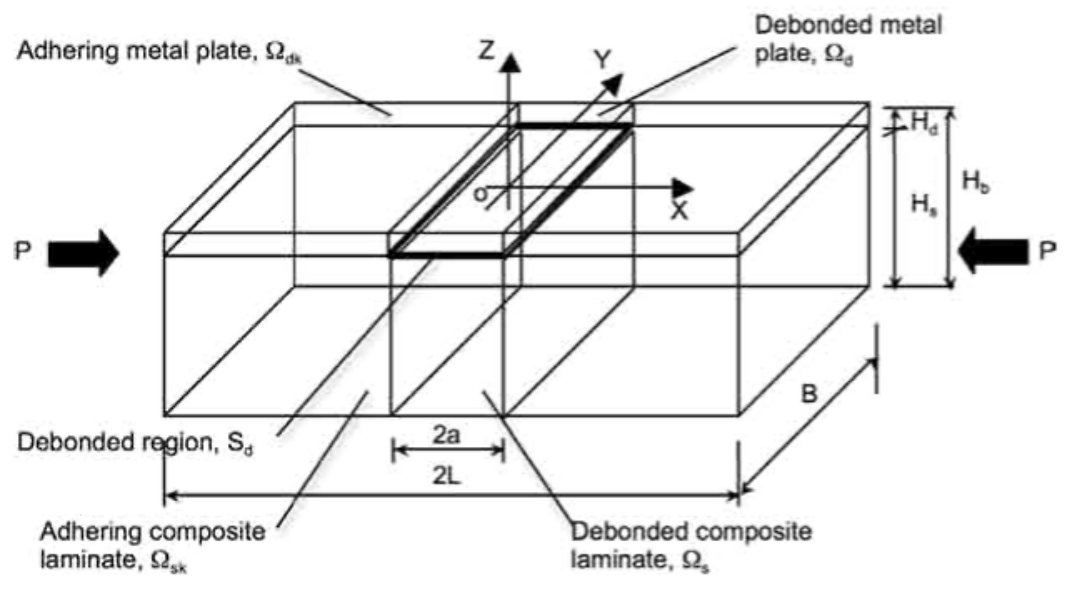

Fig. 2 Hybrid laminate with a through-thewidth debonded region, loaded in compression. interface layers of zero thickness. Inter-laminar stresses are modelled by suitable constitutive laws, which can include the effects of anisotropy, plasticity, viscosity, damage and so forth. The simplest interface model that can be imagined is probably a continuous distribution of linear elastic springs. Different values can be assumed for the elastic constants in the directions normal and tangent to the interface plane. Serizawa ${ }^{13}$ has proposed nonlinear elastic springs model in order to analyze specifically the failure mechanisms in adhesive-bonded joints. These models are known as elastic-interface models (EIM).

We used a one-dimensional model of a hybrid-laminated plate containing a through-the-width debonded area, at the composite-metal interface, and subjected to uniaxial compression. This is an approach to the situation of the upper-half hybrid plate during bending, as schematized in Fig. 2. The materials are homogeneous and linear elastic, with orthotropy axes of the composite laminate aligned with those of the fixed reference.

Provided the suitable boundary conditions are maintained on the edges, the debonded plate can be modelled as an assemblage of beam-plates, that is, plates undergoing a deformation where the only non-zero curvature is in the XZ-plane. Under the above hypotheses, the solution will not depend upon the $Y$-coordinate and therefore all calculations will refer to a plate with unit width.

For a beam-plate undergoing moderate rotations, von Kármán's plate theory can be used for the bending problem

$\frac{\partial^{4} w}{\partial X^{4}}=\frac{1}{D}\left(N^{X} \frac{\partial^{2} w}{\partial X^{2}}+\frac{\partial m^{X}}{\partial X}-f^{X} \frac{\partial w}{\partial X}+q\right)$,

while the extensional problem is described by

$\frac{\partial N^{X}}{\partial X}+f^{X}=0$.

Equations (1) and (2) must now be specified for each sub-laminate: the debonded metal plate, $\Omega_{\mathrm{d}}$ of thickness $H_{\mathrm{d}}<H_{\mathrm{b}} / 2$, contained between the delamination and the nearest external surface; the adhering metal plate, $\Omega_{\mathrm{dk}}$ of thickness $H_{\mathrm{d}}$, bonded to the composite laminate by the elastic interface; the debonded composite laminate, $\Omega_{\mathrm{s}}$ of thickness $H_{\mathrm{s}}=H_{\mathrm{b}}-H_{\mathrm{d}}$, contained between the delamination and the furthermost external surface; the adhering composite laminate, $\Omega_{\text {sk }}$ of thickness $H_{s}$, bonded to the metal plate by the elastic interface. According to the so-called thick column hypothesis, it is supposed that $D_{\mathrm{s}} \gg D_{\mathrm{d}}$. So, any transverse displacements of the composite laminate, $w_{\mathrm{s}}$ and $w_{\mathrm{sk}}$, are neglected, while the transverse displacements of the metal plate, $w_{\mathrm{d}}$ and $w_{\mathrm{dk}}$, are taken into account. Axial displacements, $u_{\mathrm{d}}, u_{\mathrm{dk}}, u_{\mathrm{s}}$ and $u_{\mathrm{sk}}$, are considered in all sub-laminates.

An exact explicit solution to the above-stated differential problem can be obtained in the simpler case, lacking tangential springs $\left(k_{\mathrm{X}}=0\right)$, leading to what is called the Winkler-interface model. In the more realistic, general case $\left(k_{\mathrm{Z}} \neq 0\right.$ and $\left.k_{\mathrm{X}} \neq 0\right)$, of the EIM, an approximate explicit solution is found by neglecting the nonlinear contribution to the axial strain. Consequently, the adhering metal plate $\Omega_{\mathrm{dk}}$ is treated as a standard beam-plate that undergoes transverse displacements, thereby acting as an elastic restraint for the debonded metal plate $\Omega_{\mathrm{d}}$, although it is not directly involved in the instability phenomenon.

Details of the solution are rather lengthy and can be found elsewhere. ${ }^{2}$ The energy release rate, $G=-\partial \Pi / \partial a$ (where $\Pi$ is the total potential energy of the system), is the sum of the contributions of modes I and II. Using the virtual crack closure technique, these are

$$
\begin{aligned}
G_{\mathrm{I}}= & \frac{1}{2} k_{\mathrm{Z}} a_{\mathrm{dk}}^{2} \frac{8 \lambda}{2 a / \lambda-\sin (2 a / \lambda)} \\
& \times\left(a+\omega \tanh \frac{L_{\mathrm{k}}}{\omega}\right) \frac{P-P^{C}}{A_{\mathrm{s}}}
\end{aligned}
$$

and

$$
G_{\mathrm{II}}=\frac{1}{2} k_{\mathrm{X}}\left(\omega \tanh \frac{L_{\mathrm{k}}}{\omega} \frac{P-P^{C}}{A_{\mathrm{s}}}\right)^{2} .
$$




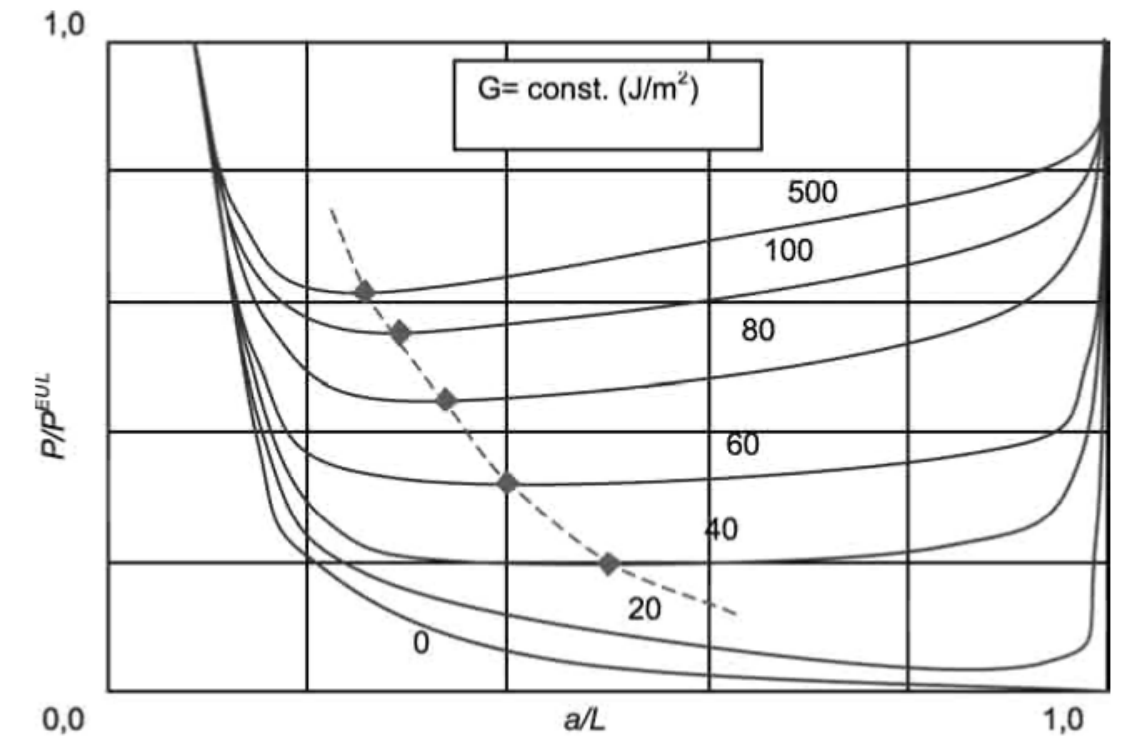

Fig. 3 Dimensionless applied load versus debonding length. Regions of stable (to the left of the dotted line) and unstable (to the right of the dotted line) crack propagation for adhesives with different fracture energies.
A conventional measure of the relative amount of mode II to mode I at the crack tip is given by the modemixity angle,

$\Psi=\arctan \frac{G_{\Pi}}{G_{\mathrm{I}}}$.

In a hybrid fibre-metal laminates, the metal sheet is bonded to the composite core by a layer of polyurethane adhesive. The elastic constants of the interface can therefore be chosen as follows:

$k_{\mathrm{Z}}=\frac{E_{\mathrm{ad}}}{t} \quad$ and $\quad k_{\mathrm{X}}=\frac{2 G_{\mathrm{ad}}}{t}$.

Figure 3 shows a contour plot of $G$ as a function of the dimensionless debonding length, $a / L$, and applied load, $P / P^{E U L}$. During the pre-critical phase, the metal sheet and composite core do not undergo any relative displacements. Thus, the inter-laminar stresses are zero and so is $G$. Consequently, no growth is predicted for loads less than the buckling load. During the post-critical phase, $G$ increases with increasing load. When the critical value is reached, debonding growth occurs and the point representing the state of the system, $(a, P)$, moves along the contour line, $G=G_{\mathrm{c}}$. Growth will be stable if $P$ increases with increasing $a$, unstable if $P$ decreases.

Dotted line in Fig. 3 represents minimum curves for every value of $G$. Contour lines are decreasing curves below a certain threshold level, $G_{\mathrm{c}}^{\text {th }}$. This is equivalent to say that growth will be always unstable, regardless of the length of the debonded region. Therefore, it is necessary not to use adhesives with $G_{\mathrm{c}}$ value below $G_{\mathrm{c}}^{\text {th }}$, or there will be a risk of delamination for any size of the debonded zone. However, for adhesives with a $G_{\mathrm{c}}$ value above the threshold, there will be a stable growth for any delamination lengths greater than the corresponding value for the minimum of the curve. For smaller lengths, the inter-facial crack will eventually arrest and no growth will take place if the applied load is not increased. This is the reason why it is so important to accurately measure the critical energy release rate of the adhesive used in manufacturing a hybrid fibre-metal laminate: it is also essential to select an adhesive with a $G_{\mathrm{c}}$ above a certain value, $G_{\mathrm{c}}^{\text {th }}$, in order to avoid unstable crack growth for every defect size, and high enough to sustain the design load safely, but not too high because the adhesive line has to be the weakest link so as to become the path for crack growth. Otherwise, the crack could divert its way towards the composite substrate.

Figure 4 shows variation of delamination lengths and applied load at constant value of the mode-mixity angle for a certain adhesive fracture energy. There is an increase of the applied load necessary for crack propagation as $\Psi$ increases, at constant value of delamination length. This is in good agreement with the experimental fact that values measured in a pure mode II test, $G_{\text {IIc }}$, are several times those measured in a pure mode I test, $G_{\mathrm{Ic}}$. At constant applied load, $\Psi$ increases when the delamination length grows, and there is consequently a tendency towards a transition from the opening to the sliding mode during the process of debonding buckling.

However, it is not an easy task to obtain reliable values of mixed-mode fracture energy of elastomeric structural adhesives, and special care has to be taken in the experimental set-up and data-reduction procedure.

\section{MODE I ADHESIVE FRACTURE ENERGY: TDCB TEST}

\section{Joint manufacture}

Metal-to-metal joints have been prepared. A total of 10 specimens have been prepared and tested for every 


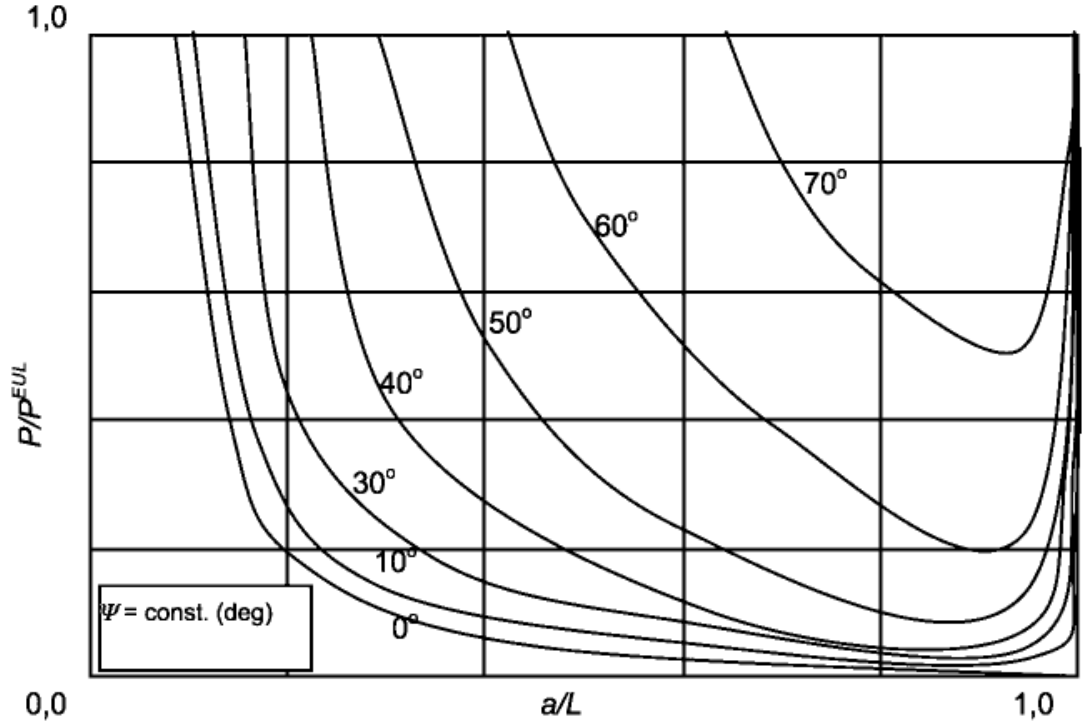

Fig. 4 Influence of mode-mixity angle on dimensionless applied load versus debonding length curves. experimental point, that is, for every value of mode mixity angle (six angles have been tested, ranging from $0^{\circ}$, peel, to $90^{\circ}$, shear). Duplicate test results have been considered suspicious if they differ by more than $10 \%$. Only specimens with a cohesive failure have been considered valid, and no less than five valid values have been used to obtain the $95 \%$ confidence limits, all in accordance with ASTM D3433. To make the TDCB test specimens, a profile with a ' $U$ ' section has been shaped by bending a mild steel metal sheet $1.5 \mathrm{~mm}$ thick. They were abraded with 180-mesh alumina grit and cleaned with acetone. The metal sheets were further cleaned with an organic vapour solvent (1,1,1-trichloro-ethylene) and surface treated by immersion in hot phosphoric acid. A primer was applied to both surfaces, following the recommendations of the adhesive manufacturer, adhesive was evenly spread across the surfaces and parts were joined with the adhesive to get an 'H' shape.

The adhesive used was bi-component polyurethane (SikaForce ${ }^{\circledR} 1897$ 2C) with a wide elastic deformation range. Placing steel wires of same diameter in between both parts assures a bond-line thickness of $1 \mathrm{~mm}$. A pressure of $6 \mathrm{kN} / \mathrm{m}^{2}$ has been applied during curing by imposing a certain weight on the joint. At the end where the load is applied, a PTFE film $15 \mu \mathrm{m}$ thick was placed in order to act as a crack starter. The joint assembly was cured at room temperature for at least $24 \mathrm{~h}$. The specimens were placed in the tapered stainless steel jigs and secured with bolts (see Fig. 5). Care has been taken to control variations in bond-line thickness. To maintain the constant bond-line thickness, steel wire of $1 \mathrm{~mm}$ in diameter was inserted into the ends of the specimen prior to forming the joint. The bond-line edges were covered with a thin layer of white correction fluid in order to obtain a good contrast that will allow an easy follow-up on the grow-

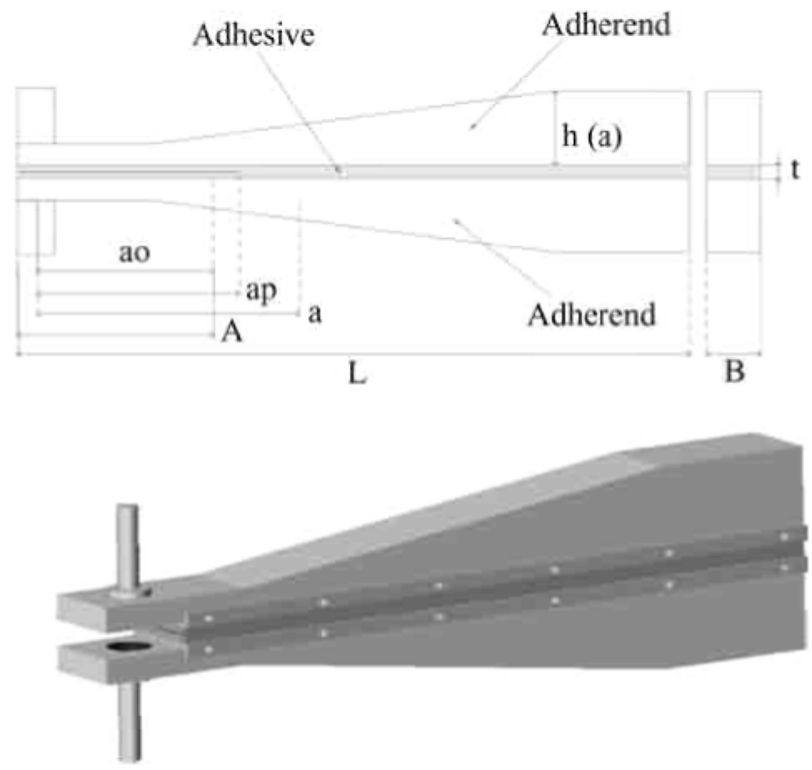

Fig. 5 Tapered double cantilever beam (TDCB) test specimen and modified testing fixture for adhesively bonded joints (see Table 1 for dimensions.)

ing of the crack, and ease crack length measurements. Table 1 shows the details of the adhesive joint specimens manufactured.

\section{Joint testing}

Tests were performed following Protocol BS 7991-2001, ${ }^{8}$ with a constant cross-head displacement rate of $0.1 \mathrm{~mm} / \mathrm{s}$. Simultaneous values of applied load, displacement and crack length were measured as far as the crack was growing inside the tapered section of the test specimen, 
Table 1 Details of the adhesive joint system in TDCB and DMMB tests

\begin{tabular}{lll}
\hline Test specimen & TDCB & DMMB \\
\hline Materials & Mild steel/mild steel, U profile & GRP/mild steel, flat sheet \\
Test fixture & Stainless steel, tapered with $m=2633 \mathrm{~m}^{-1}$ & Stainless steel \\
Width of joint, $B$ & $25 \mathrm{~mm}$ & $25 \mathrm{~mm}$ \\
Thickness of GRP substrate arm, $H_{2}$ & - & $5.0 \mathrm{~mm}$ \\
Thickness of steel substrate arm, $H_{1}$ & $1.5 \mathrm{~mm}$ & $1.5 \mathrm{~mm}$ \\
Thickness of adhesive layer, $t$ & $1 \mathrm{~mm}$ & $1 \mathrm{~mm}$ \\
GFP substrate axial modulus & - & $17 \mathrm{GPa}$ \\
Steel substrate axial modulus & $207 \mathrm{GPa}$ & $207 \mathrm{GPa}$ \\
\hline
\end{tabular}

obtaining the biggest possible number of experimental measurements from each test specimen. The test was stopped after every crack propagation. The crack was allowed to grow until full arrest. Both propagation and arrest loads were used for data reduction. The position of the crack tip was derived with precision using two microscopes that move in parallel with the bond line. Two video cameras were incorporated in order to watch the images on a computer screen and to record the experiment for further analysis.

After crack has propagated (displacement control) and the load has levelled off, the specimen is fully unloaded and any permanent offset displacement on the loading trace is considered as an indication of plastic deformation of the substrates. Subsequently, the specimen is reloaded until a new propagation event is observed. This operation is not specified in the test protocol, which only requires unloading whenever crack tip has overgrown the tapered section and before the final failure of the joint, with the purpose of assessing that LEFM assumptions have not been violated, which means that no substrate plastic deformation can be observed. As intended, it is necessary to completely unload and fully reload after every propagation in order to measure the variation of compliance in the test fixture throughout the test. No plastic deformation of the adherends in any case was observed.

\section{MODE I+II ADHESIVE FRACTURE ENERGY: DMMB TEST}

\section{Joint manufacture}

Surface treatment for the metal sheet was the same as previously described for the TDCB specimen. Also the adhesive system, the application procedure and thickness were the same, as already mentioned. Regarding the GFR substrate, vynilester matrix (Dion 9102-500, Reichhold Durham NC 27703, USA) and glass-fibre woven fabrics $\left(800 \mathrm{~g} / \mathrm{m}^{2}\right.$ aerial weight, SP Gurit, Zürich, Switzerland) as reinforcement were used. Test specimens were prepared and tested in an atmosphere maintained at $50 \pm 4 \%$ rela-

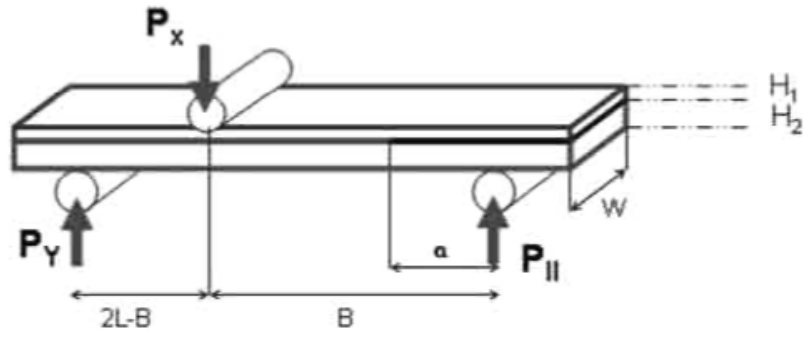

Fig. 6 Dissimilar mixed-mode bending (DMMB) test specimen. Thickness ratio, $H_{1} / H_{2}$, is kept constant and mode mixity angle is varied modifying $B$ value,

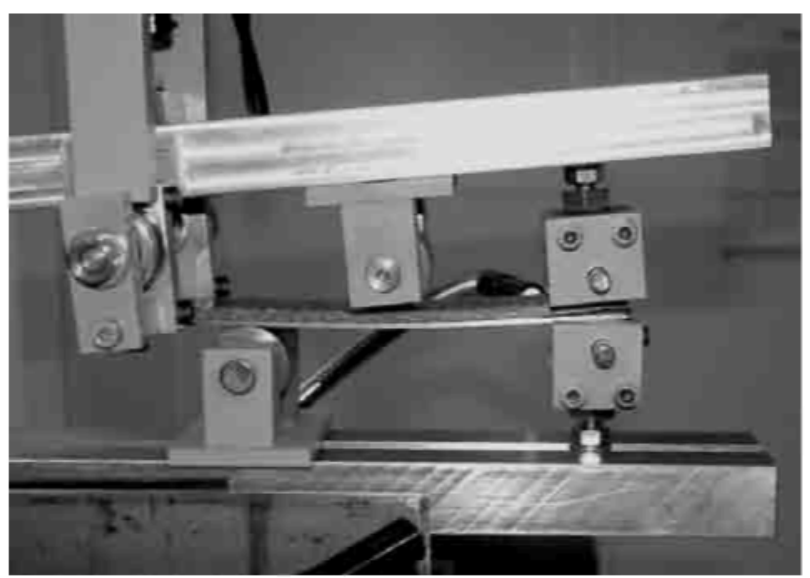

Fig. 7 DMMB testing rig with the specimen inserted. GFR is on top and metal substrate on bottom.

tive humidity and $23 \pm 1{ }^{\circ} \mathrm{C}$. GFR substrates were abraded with $180 / 220$ mesh alumina grit. The ratio of the composite arm thickness to the metal sheet thickness was kept constant for all the specimens (see Fig. 6 and Table 1 for the physical dimensions of the specimen).

The rig was made of steel, flat sheet adherends, and the system of load transmission was modified in order to reduce the effects of the rig own weight on the load applied to the specimen (see Fig. 7). For the testing, a Tecnitest electro-static testing machine was used equipped with a 


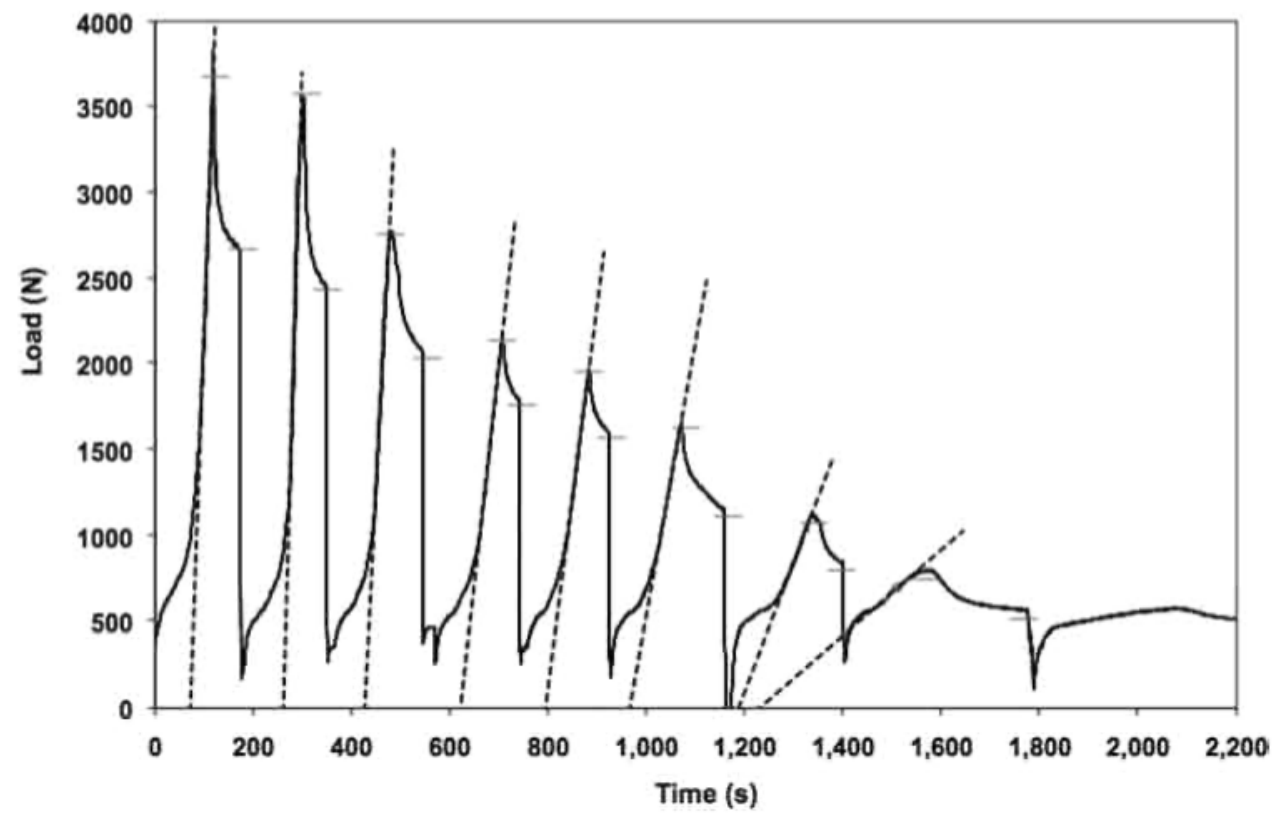

Fig. 8 A typical load-time trace for a TDCB test.

load cell of $5 \mathrm{kN}$. Also a digital video device was used in order to identify the crack length per each level of applied load.

\section{Joint testing}

The DMMB test, introduced by Soboyejo et al.,$^{14}$ was extended to hybrid metal-GRP laminae by G.V. Marannano, A. Pasta ${ }^{15}$ as a more flexible version of the MMB test configuration, because it allows the possibility to obtain mixed-mode loading ratios by just changing the rig setting and avoiding the need to use different thickness ratios in the specimens. A constant $H_{1} / H_{2}$ ratio has been used, for equal bending stiffness of both substrates. There is not an equivalent protocol to assess the DMMB testing as the BS 7991-2001 used for the TDCB testing. However, the procedure for DMMB testing was kept as close as possible to that used for TDCB testing in every experimental detail: cross-head displacement rate, loading and unloading after every crack propagation, data acquisition and crack location.

\section{RESULTS AND DISCUSSION}

\section{TDCB test data reduction}

Figure 8 shows a typical load versus time curve that was measured during a TDCB test. The first peak corresponds to the propagation of the pre-crack that started in the adhesive because of the inclusion of the PTFE film. Data from the first propagation have been discarded as the crack tip does not physically behave as the real crack observed in service. The last propagation has not been considered since the crack tip was already out of the tapered area of the test fixture.

Figure 9 shows a load versus displacement trace of all the load-unload-load cycles in a typical test. For the sake of clarity, unload traces have been disregarded and the slopes in the area of proportional behaviour have been determined. As can be observed, a deformation at almost constant load for every load cycle appears. It is related to the deformation of the elastomeric adhesive mainly. It follows a transition zone until a proportional deformation is reached, which corresponds to the elastic deformation of the metal adherents. Finally, the trace starts separating from proportionality until the propagation of the crack is initiated. Straight lines are fitted to the proportional behaviour curves and extrapolated. It can also be observed how all of them cut in a single point, establishing the limit to which the adhesive bears most of the deformation. Passing this point, the metallic fixture starts deforming and storing elastic strain energy.

Mostovoy $^{7}$ has been the first to propose TDCB test specimen to measure the plane-strain fracture toughness in adhesives. The different methods of analysis gathered in the 'British Standard' basically come from the equation of Irwin-Kies, where the energy of adhesive fracture, $G_{\mathrm{Ic}}$, is obtained from the following equation:

$G_{\mathrm{Ic}}=\frac{P^{2}}{2 B} \frac{d C}{d a}$,

where $P$ is the applied load, $B$ is the width of the adhesive joint, $C$ is the compliance and $a$ is the length of the crack. 


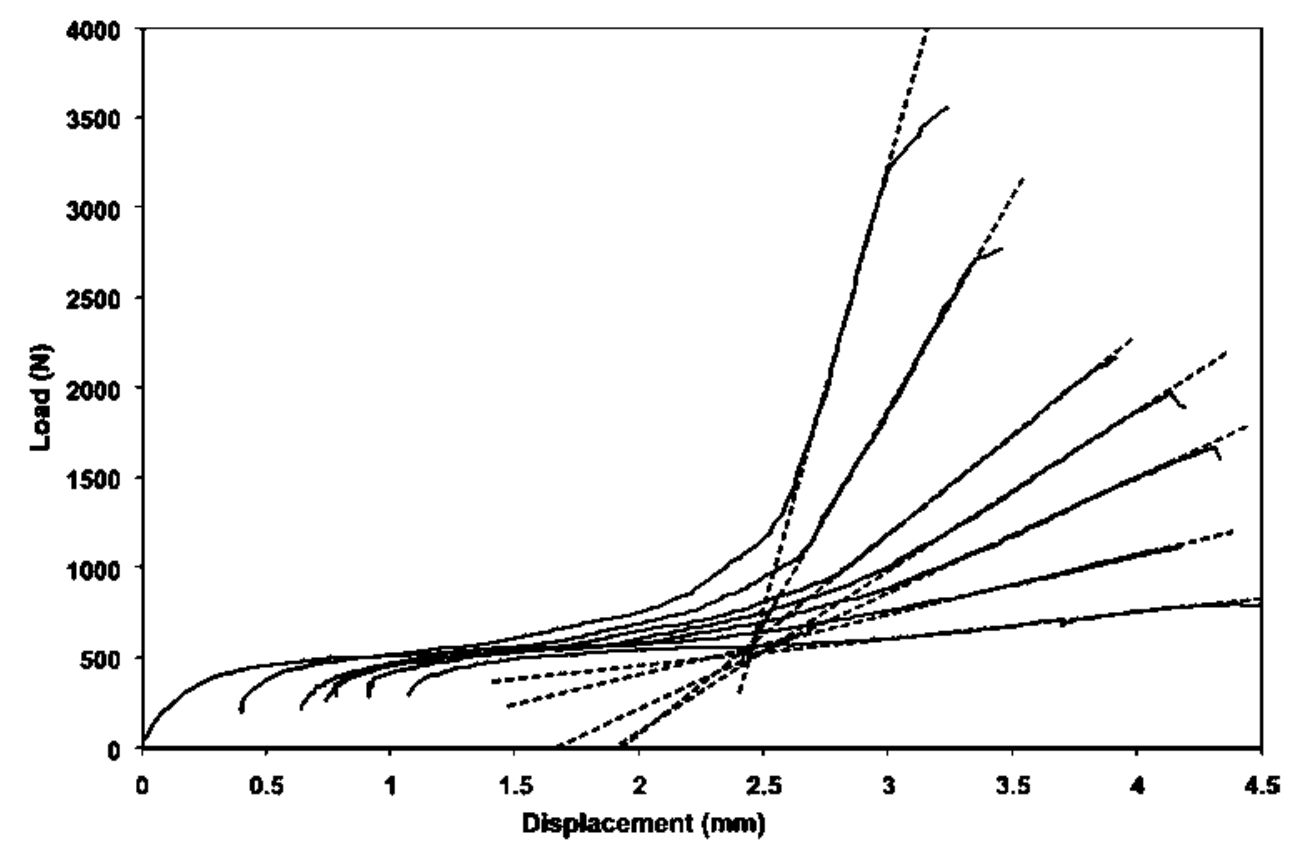

Fig. 9 A typical load-displacement trace for a TDCB test.

The difference between different methods of analysis lies on the manner of obtaining $d C / d a$. The most straightforward approach is the application of a simple beam theory that considers contribution from bending and shear deflections. In such case, the compliance of the beam, $C$, is given as,

$C=\frac{8\left(a^{3}+b^{2} a\right)}{E_{\mathrm{s}} B b^{3}}$,

where $b$ is the height and $E$ is the Young's modulus of the metal in which the testing fixture has been made of. This equation is valid only for thin layers of adhesive. $E_{s}$, the elastic modulus of the substrate, was determined independently in a tensile standard test, using a conveniently sensitive extensometer.

Differentiating Eq. (8) and substituting it in Eq. (7), the equation below is derived:

$G_{\mathrm{Ic}}=\frac{4 P^{2}}{E_{\mathrm{s}} B^{2}}\left(\frac{3 a^{2}}{b^{3}}+\frac{1}{b}\right)=\frac{4 P^{2}}{E_{\mathrm{s}} B^{2}} m$.

Beams were manufactured using a $\mathrm{CNC}$ milling machine to produce a constant geometry factor, $m$. Metrological control of the specimens was performed to guarantee a precision of $\pm 1 \%$ in every dimension.

$m=\frac{3 a^{2}}{b^{3}}+\frac{1}{b}$.

In this study, the value of $m$ has been chosen as $2633 \mathrm{~m}^{-1}$, taking into account that the value of $b$ in every section is equal to the height of the tapered fixture plus $1.5 \mathrm{~mm}$ of metal sheet. In such manner, $G_{\text {Ic }}$ value should be independent to the length of the crack.

As mentioned, using the corrected beam theory to introduce the contributions due to deflection and rotation of the beam root, the analysis can be further refined. Such effects could be more important than the mentioned owing to shear deformation when calculating $G_{\mathrm{Ic}}$ using TDCB test specimens made of metal substrates. According to Blackman, ${ }^{3}$ an analytical expression has been derived for compliance that includes previous corrections.

$C=\frac{8 m}{E_{s} B}\left[a+0.64\left(\frac{3}{m}\right)^{1 / 3} a^{2 / 3}-\frac{2}{3} x_{0}\right]$.

Using this equation in Eq. (7), a new expression for $G_{\mathrm{Ic}}$ is derived,

$G_{\mathrm{Ic}}=\frac{4 P^{2} m}{E_{\mathrm{s}} B^{2}}\left[1+0.43\left(\frac{3}{m a}\right)^{1 / 3}\right]$.

Figure 10 shows values obtained from experimental measurements using Eq. (9). Crack propagation and crack arrest loads have both been taken into consideration. It is clear that values of $G_{\mathrm{Ic}}$ (both $G_{\mathrm{Ici}}$, at initiation, and $G_{\mathrm{Ica}}$, at arrest) have not resulted to be independent to the size of the crack.

Compliance $(C)$ as derived by the beam theories gathered in the British Standard ${ }^{8}$ does not reflect real behaviour of the test device because some of the assumptions of these theories do not stand as reasonable for our particular adhesive system. Particularly, adhesive layers are quite thick and polymer shows a highly nonlinear behaviour. Other 


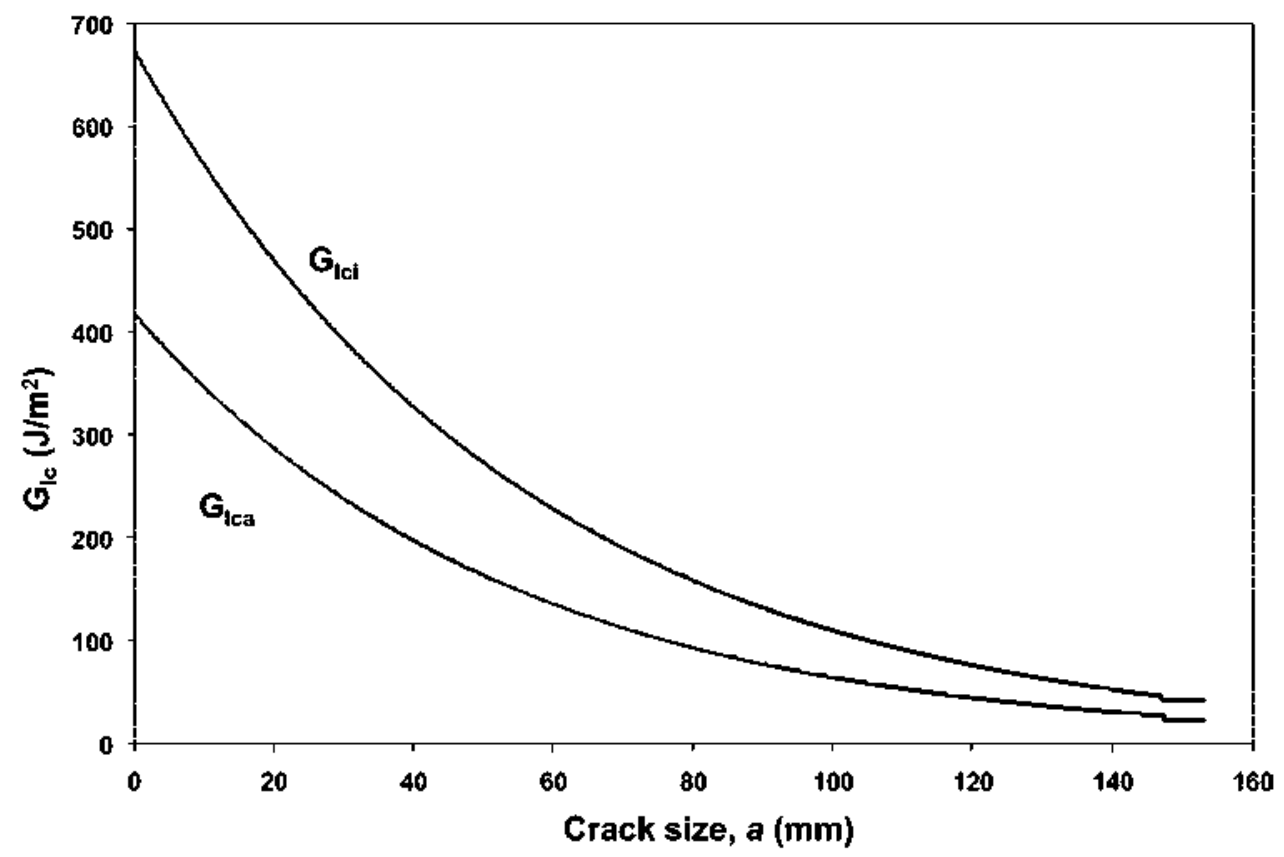

Fig. 10 Adhesive fracture energy versus crack length (resistance curve) as deduced from analysis techniques, for crack propagation onset $\left(G_{\mathrm{Ici}}\right)$ and crack arrest $\left(G_{\mathrm{ICa}}\right)$ loads.

more elaborated theories that take into account rotation effects in the root of the beam, which are included in the 'British Standard', do not correct the detected problem either. Some of the assumptions are still not being complied because the 'British Standard' is meant for rigid adhesives with linear behaviour until breakage and it is only valid for adhesives in very thin layers. Such case does not match our study, as the elastomeric adhesive shows a nonlinear behaviour in all the strain range. Moreover, it is also too thick.

It is thereby necessary to perform the test using a method also included in BS 7991-2001, and determine compliance variations throughout the test. Compliance for each length of the crack is measured from the slope of straight lines on Fig. 9. Results have been obtained from the unload-reload loop for every crack growth event. Figure 11 shows the variation of $C$ with respect to the crack size, $a$. Compliance closely fit to a power law,

$C=3.10^{-7} e^{18.05 i a}$

From Eq. (13), $d C / d a$ curves can be obtained throughout the test. Using this experimental measured $d C / d a$, adhesive fracture energy, $G_{\text {Ici }}$ and $G_{\text {Ica }}$, for every propagation event can be determined from Eq. (7). The results are shown in Fig. 12. The least-squares fit shows now that adhesive fracture energy is indeed independent to the crack size, as had been expected. Although both values, $G_{\mathrm{Ici}}$ and $G_{\text {Ica }}$, have been included in the graph, the smallest value is taken for design purposes, which refers to the value obtained from the load at the crack arrest. The average value of the adhesive fracture energy is $G_{\mathrm{Ica}}=5407 \mathrm{~m}^{-2}$ for the adhesive system used.

Figure 9 shows that for every load cycle, there is an initial deformation due to the strain taken mostly by the elastomeric adhesive. The adhesive itself is storing an elastic strain energy that is not available for crack growth. In consequence, this energy is not taken into account when calculating $G_{\mathrm{I}}$, because adhesive is not capable of releasing that energy as the inter-facial crack tip propagates. The reason is the viscoelastic behaviour of the adhesive, with a relaxation time longer than the crack growth rate. Elastic strain energy stored in the stainless steel tapered fixture is the only energy readily available to create new fracture surface, as it is immediately released at the crack growth onset. The steel test fixture start deforming and storing a non-negligible share of the energy when the adhesive reaches its limit of deformation, and then starts strengthening. This precisely takes place at the very point where all straight lines cut. From that point on, elastic linear behaviour is due to the deformation of the steel test fixture plus the deformation of the adhesive itself.

\section{$D M M B$ test data reduction}

The inter-laminar crack growth behaviour was analyzed by performing a $D M M B$ test, with a delamination introduced within the interface between the metal and the composite during the manufacturing stage, and placed as shown in Fig. 6, which was proposed by Soboyejo ${ }^{14}$ to study the onset and growth of delamination for a wide 


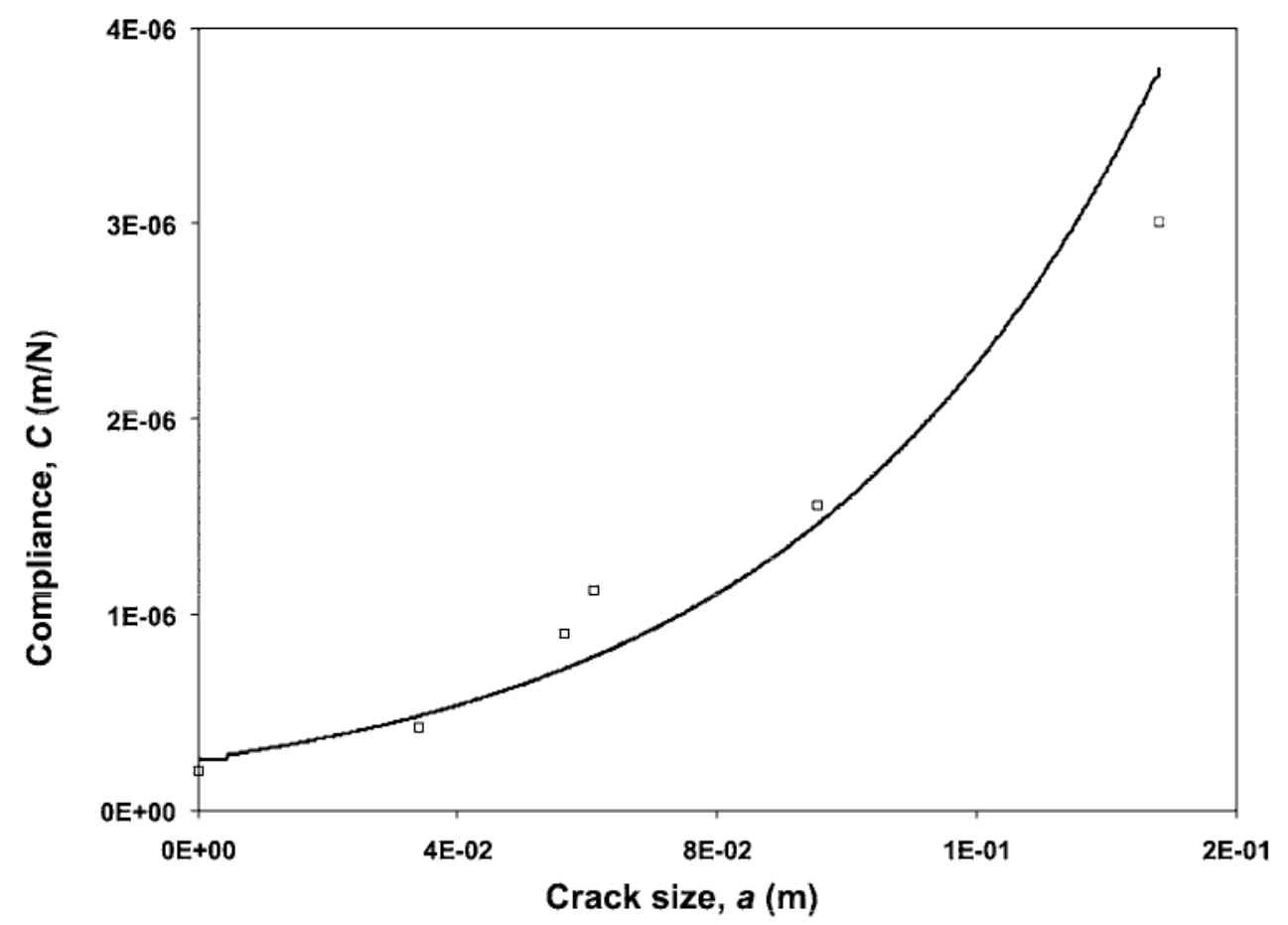

Fig. 11 Measured compliance versus crack length.

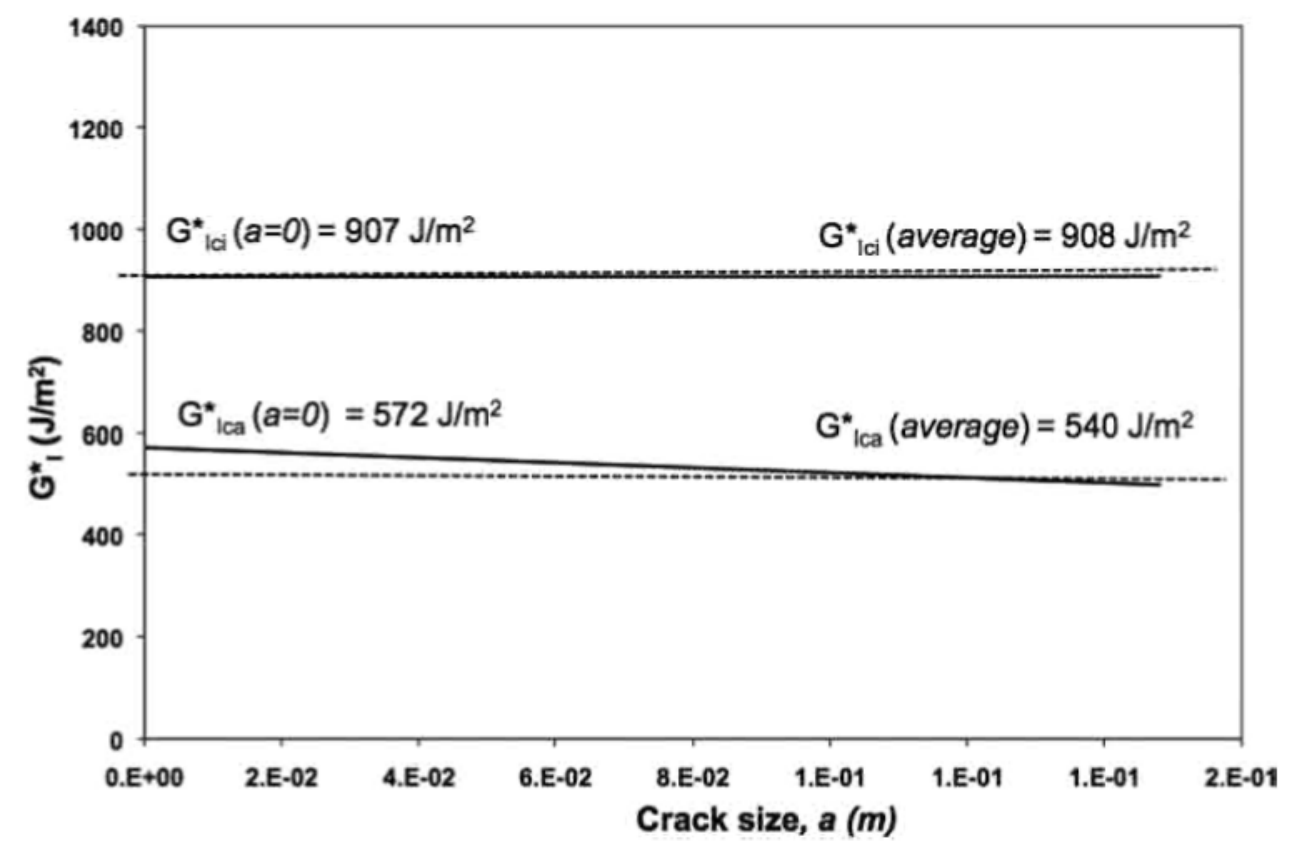

Fig. 12 Adhesive fracture energy versus crack length (resistance curve) as deduced from measured compliance, for crack propagation onset $\left(G_{\text {Ici }}\right)$ and crack arrest $\left(G_{\text {Ica }}\right)$ loads.

range of mixed-mode loading ratios. The DMMB test is such to introduce a mixed-mode (modes I and II) loading ratio that can be easily varied by modifying the lever length of the loading rig (i.e. varying the length $B$ in Fig. 6). The same ratio can also be varied by modifying thicknesses $H_{1}$ and $H_{2}$ of the two cantilever arms sepa- rated by the inter-facial crack although this generates an asymmetric laminate $\left(H_{2} / H_{1} \neq 1\right)$.

Figure 13 shows a schematic representation of the DMMB loading set-up, highlighting the role of the external forces applied to the specimen and responsible for the onset of loading modes I and II. Figure 14 shows 


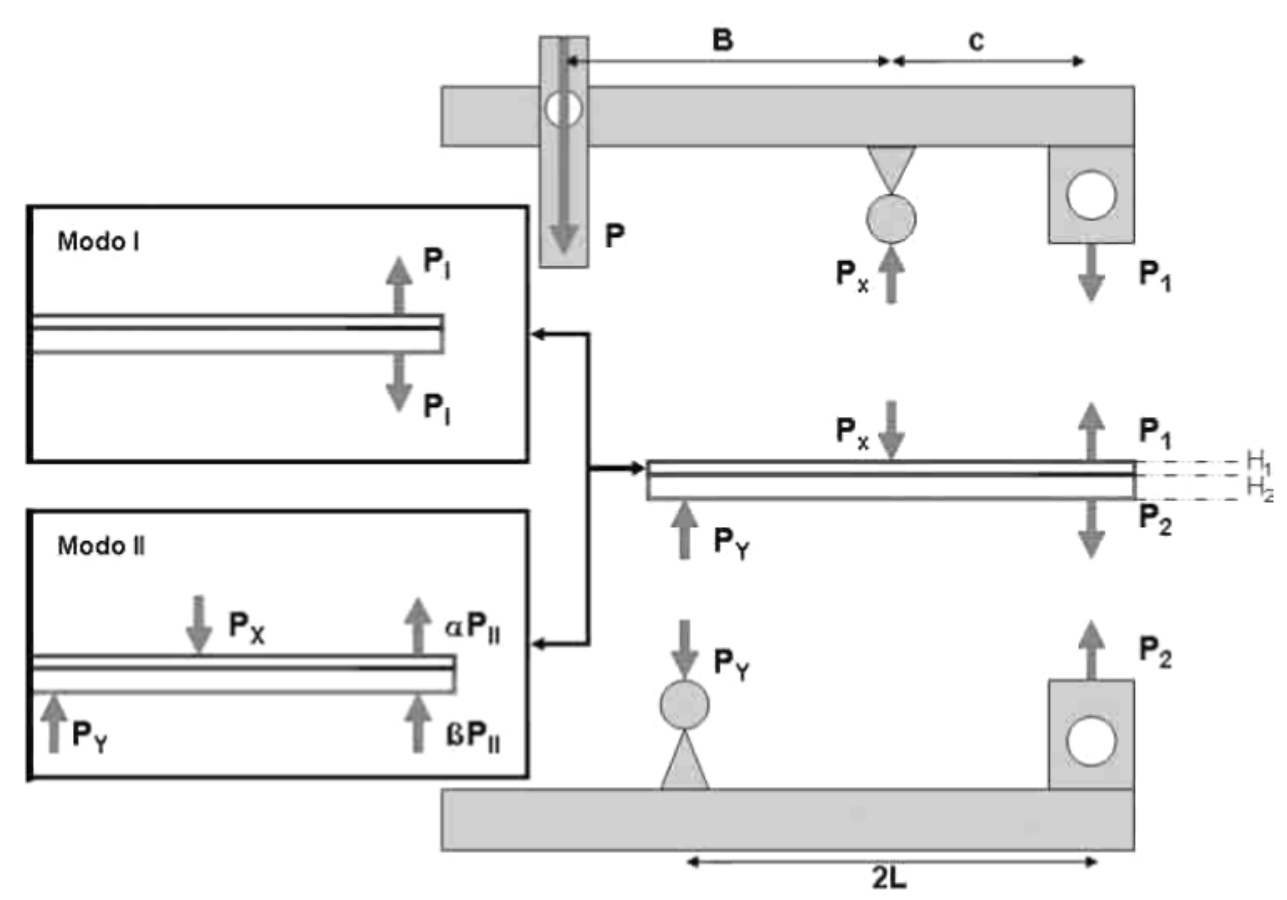

Fig. 13 Schematic representation of the DMMB loading set-up, with contribution of the applied loads to modes I and II.

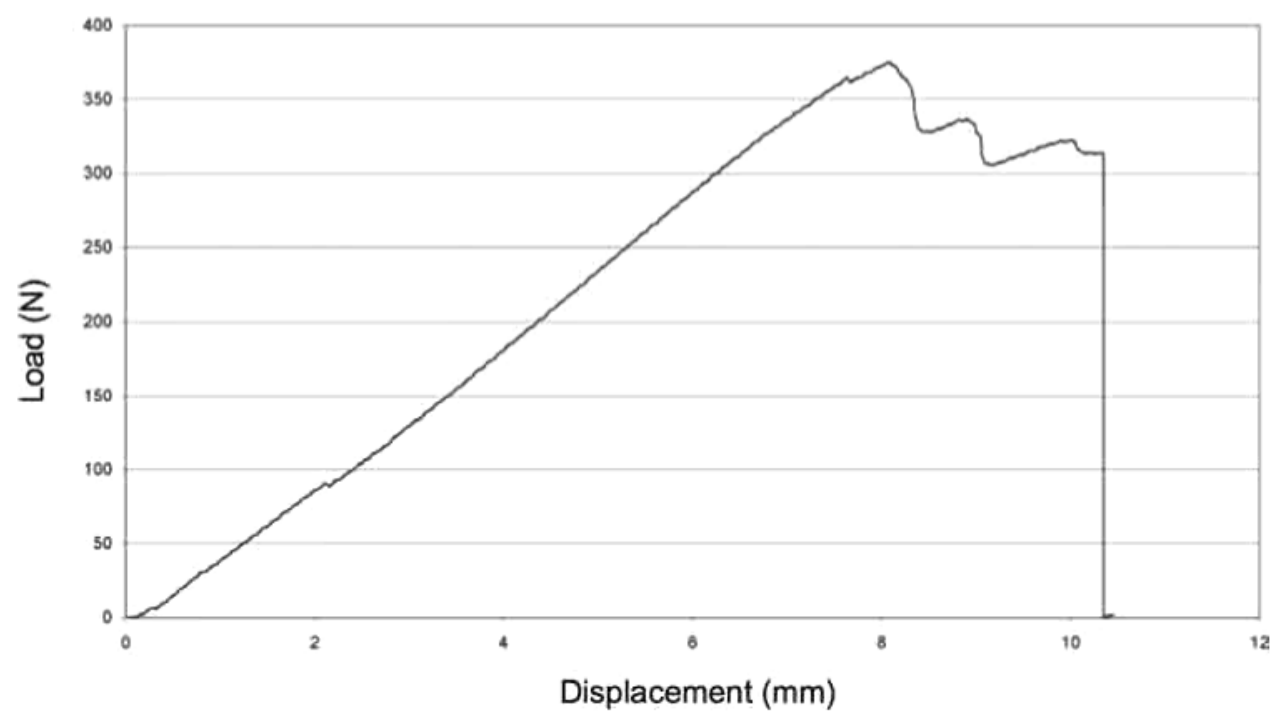

Fig. 14 Load versus displacement plot for a hybrid material.

a load versus displacement trace in a typical test. It is possible to identify the points at which debonding starts growing and the compliance variation due to the extended crack. This information is used so as to evaluate total strain energy release rate at the crack tip in the same way that was followed with TDCB testing. From Fig. 13, by imposing equilibrium on the upper loading lever, external forces $P_{1}$ and $P_{\mathrm{x}}$ as functions of the driving external force $P$ can be determined. By imposing equilibrium of the sample, $P_{2}$ and $P_{y}$ can be considered as functions of $P$ and of the system geometry. The two sets of forces associated with pure modes I and II must give same external forces due to their mutual superposition. Considering also that $\alpha$ and $\beta$ give fractions of force $P_{\mathrm{II}}$ acting in each sample arm, and hence $\alpha+\beta=1, P_{\mathrm{I}}$ and $P_{\mathrm{II}}$ can be determined as functions of $P$ and system geometry $^{15}$

$P_{\mathrm{I}}=\frac{P c}{B}-\frac{\alpha P(B+c)}{\alpha+\beta}\left(\frac{1}{B}-\frac{1}{2 L}\right)$ 


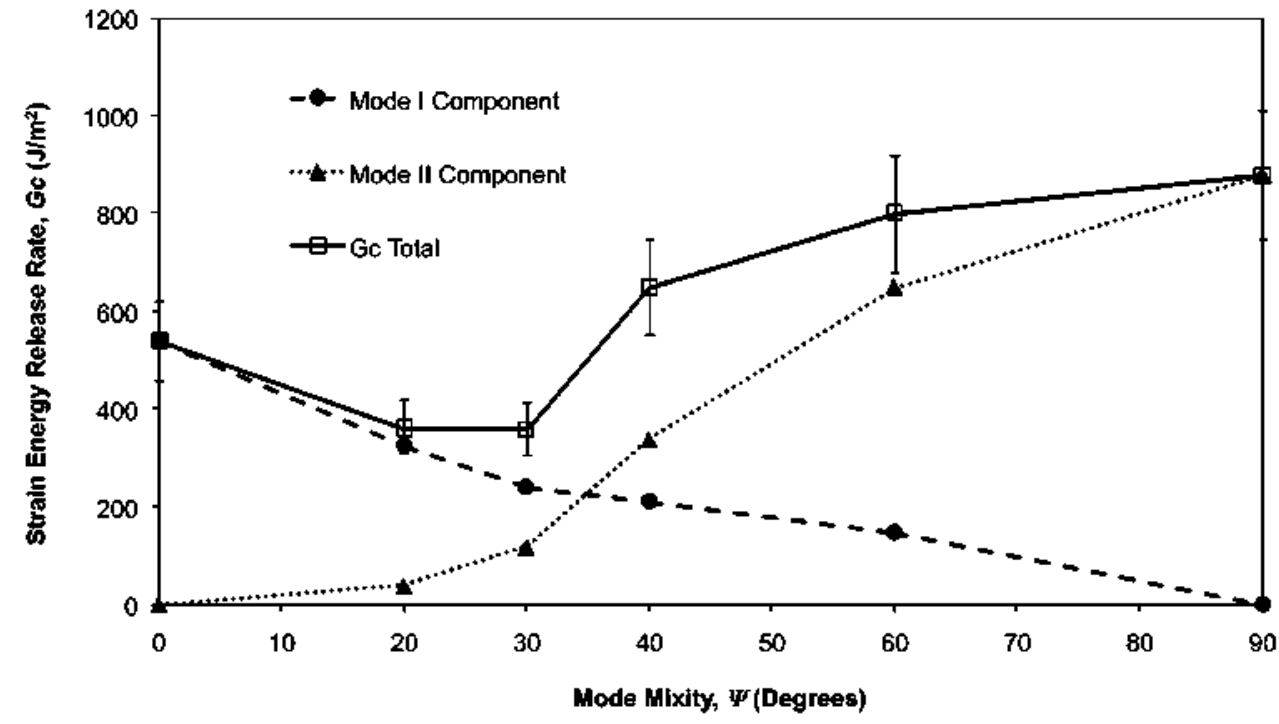

Fig. 15 Variation of strain energy release rate with mode mixity.

and

$P_{\mathrm{II}}=\frac{P(B+i)}{\alpha+\beta}\left(\frac{1}{B}-\frac{1}{2 L}\right)$.

Mode I strain energy release rate for crack growth can be written as

$G_{\mathrm{I}}=\frac{P_{\mathrm{I}}^{2}}{2 W} \frac{\partial C}{\partial a}$

where $C$ is sample compliance. If there is no crack growth, displacement $v$ is proportional to the load, $v=C . P_{\mathrm{I}}$, so that Eq. (16) becomes

$G_{\mathrm{I}}=\frac{P_{\mathrm{I}}^{2}}{2 W} \frac{\partial\left(\frac{v}{P_{I}}\right)}{\partial a}$

The principle of virtual work can be adopted and extended to the case of two different material lavers with growing delamination crack at their interface, and thickness in different arms so as to derive relative displacement v of load application points. Substituting Eq. (17)

$G_{\mathrm{I}}=\frac{6 P_{\mathrm{I}}^{2}(a+\chi b)^{2}}{W} \sum_{j=1}^{2} \frac{1}{W E_{\mathrm{i} 1} H_{j}^{3}}$

in which $\chi$ is the crack length correction for crack tip rotation as defined elsewhere. ${ }^{16}$

Mode $\Pi$ strain energy release rate can be adapted from $\mathrm{Eq} .(17)$, where displacement $v$ has now become a more complex definition:

$v=v_{1}+v_{2}+v_{3}$,

with $v_{1}$ representing virtual displacements for $0<x<a$, $v_{2}$ for $a<x<B$, and $v_{3}$ for $B<x<2 L$ (see also Fig. 6). The principal of virtual works can be adopted again to determine these displacements. The resulting relation for $G_{\text {II }}$ is then

$$
\begin{aligned}
G_{\mathrm{II}}= & \frac{3}{2}\left[\frac{P a(B+c)(2 L-B)}{B W L}\right]^{2} \\
& \times\left(\frac{\alpha^{2}}{E_{11} H_{1}^{3}}+\frac{\beta^{2}}{E_{21} H_{2}^{3}}-\frac{1}{H^{2}\left(H_{1} E_{11}+H_{2} E_{21}\right)}\right) .
\end{aligned}
$$

To achieve a pure mode II loading, action of forces $\alpha P_{\mathrm{II}}$ and $\beta P_{\mathrm{II}}$ needs to produce the same deflection of two delamination arms, that is, the two arms need to have the same curvature. ${ }^{16}$ This condition can be analytically expressed by equations below, valid for arms having generally different thicknesses and elastic moduli (i.e. different materials):

$\alpha=\frac{E_{11} H_{1}^{3}}{E_{11} H_{1}^{3}+E_{21} H_{2}^{3}}$

Total strain energy release rate is obtained by applying the principle of linear superposition

$G=G_{\mathrm{I}}+G_{\mathrm{II}}$

Figure 15 shows variation of $G_{\mathrm{c}}$ components with mode mixity. $G_{\text {Ic }}$ decreases as the mixity angle increases, $\mathrm{Eq}_{\mathrm{q}}(5)$, while $G_{\text {II }}$ increases as the mixity angle does. However, from $\mathrm{Eq} .(22)$, the total strain energy release rate shows a minimum for approximately $\Psi=30^{\circ}$.

\section{CONCLUSIONS}

An elastic-interface model for debonding buckling of hybrid fibre-metal laminates has illustrated the role 
portrayed by geometric and elastic parameters. The process of debonding growth has been discussed briefly. Contours plots of $G$ markedly reveal different qualitative trends regarding inter-facial crack growth and stability, depending on which mode has the preponderant weight in growth criterion. The role played by mode-mixity angle, $\Psi$, shows a transition from mode I to mode $\Pi$ in the debonding buckling process. Proper calculation for $\Psi$ is a crucial for calculating accurate prediction of inter-facial crack growth, and shear stress that can steer debondings.

Adhesive fracture energy of elastomeric adhesives has been measured using TDBC and DMMB test specimens. Results show that $G_{c}$ decreases with crack length when data are reduced using approaches included in BS 79912001. Beam theories are invalid for deriving variations of compliance with respect to crack size due to nonlinear behaviour and great thickness of the elastomeric adhesive used in this study. However, compliance variation can be measured from the tests by unloading and reloading every time crack propagation is detected. From this information, proper values for $G_{c}$ can be determined, which is a constant value for every crack length, as should be expected.

Results obtained from DMMB testing show a variation of strain energy release rate components with modemixity angle, $\Psi . G_{\mathrm{Ic}}$ decreases as the mixity angle increases, while $G_{\mathrm{IIc}}$ increases as the mixity angle does. The total strain energy release rate shows a minimum for approximately $\Psi=30^{\circ}$.

\section{Acknowledgements}

We thank the support from Universidad Politécnica de Madrid through grant AM0402. Our gratitude to the laboratory technical staff: Mr. José Illescas, Ms. Ana Soria and Ms. Ana García for their assistance in the completion of these experiments.

\section{REFERENCES}

1 Suárez, J. C., Miguel, S., Diez de UTzurrun, I., Pinilla, P., Herreros, M. A and López, F. (2005) Material húbrido estructural para Construcción Naval: MaLECoN. In: Proceedings VT Congreso Nacional de Materiales Compuestos, Valencia.

2 Bennati, S. and Valvo, P. S. (2002) An elascic-interface model for delamination buckling in laminated plates. In: Key
Engineering Materials, Vols. 221-222 (Edited by P. Priolo), Trans Tech Publications, Switzerland, pp. 293-306.

3 Blackman, B. R. K., Hadavinia, H., Kinloch, A. J., Paraschi, $M$. and Williams, J. G. (2003) The calculations of adhesive fracture energies in mode $I$ : revisiting the tapered double cantilever beam (TDCB) test. Engineering Fracture Mecbanics 70, 233-248.

4 Blackman, B. R. K., Kinloch, A. J., Paraschi, M. and Teo, W S. (2003) Measuring the mode I adhesive fracture energy, $\mathrm{G}_{\mathrm{IC}}$, of structural adhesives joints: the results of an international round-robin. Int. F. Adbes. Adbes. 23, 293-305.

5 ASTM, ASTM D 3433. (1990) Annual book of ASTM standards. Adhesive secion 15. Filadeltia.

6 Blackman, B. R. K. and Kinloch, A. J. (2001) Fracture tests on structural adhesive joints. In: Fracture Mechanics Testing Metbods for Polymers, Adbesices and Composites (Edited by D. R. Moore, A. Pavan and J. G. Willians), Elsevier Science, Amsterdam, pp. 225-267.

7 Mostovoy, S., Crosley, P. B. and Ripling, E. J. (1967) Use of crack-line loaded specimens for measuring plane-strain fracture toughness. 7. Mater. 2(3), 661-681.

8 BSI British Standards (2001) Determination of the mode I adhesive fracture energy, $G_{I C}$, of stuctural adhesives using the double cantilever beam (DCB) and the tapered double cantilever beam (TDCB) specimens. BS 7991-2001.

9 Kachanov, L. M. (1977) Separation failure of composite materials. Pobrmer Metb. 12, 812-824.

10 Chai, H., Babcock, C. D. and Knauss, W. G. (1981) One dimensional modeling of failure in laminated plates by delamination buckling. Int. 7. Solids Strutt. 17, 1069 1082.

11 Panagiotopoulos, P. D. and Stavroulakis, G. E. (1990) The delamination effect in laminated von Kármán plates under unilateral boundary conditions. A variational-hemivariational inequality approach. 7. E/ast. 23, 69-89.

13 Serizawa, H. and Murakawa, H. (2003) Development of new interface potentials for simulating mode I \& mode II fracture behavior. Trans. 7URI 32(2), 349-354.

14 Soboyejo, W. O., Lu, G. Y., Chengalva S., Zhang, I. and Kenner, V. (1999) A modified mixed-mode bending specimen for the interfacial fracture testing of dissimilar materials. Futigue Fract. Eng. Mater. Strutt. 22, 799-810.

15 Marannano, G. V. and Pasta, A. (2007) An analysis of interface delamination mechanisns in orthotropic and hybrid fiber-metal composite laminates. Eng. Fract. Mecb. 74, $612-626$.

16 Ducept, F., Gamby, D. and Davies, P. (1999) A mixed-mode failure criterion derived from tests on symmetric and asynmetric specimens. Compos. Sci. Tecbnol. 59, 609-619.

12 Högberg, J. L., Sørensen, B. F. and Stigh, U. (2007) Constitutive behaviour of mixed mode loaded adhesive layer. Int. 7. Solids Struct. 44, 8335-8354. 